Revue d'histoire de l'Amérique française

सES REVUE D.HISTOIRE DE L'AMÉRIQUE FRANÇAISE

\title{
Étude critique du recensement du Canada de 1765
}

\section{Yves Landry}

Volume 29, numéro 3, décembre 1975

URI : https://id.erudit.org/iderudit/303462ar

DOI : https://doi.org/10.7202/303462ar

Aller au sommaire du numéro

Éditeur(s)

Institut d'histoire de l'Amérique française

ISSN

0035-2357 (imprimé)

1492-1383 (numérique)

Découvrir la revue

Citer cet article

Landry, Y. (1975). Étude critique du recensement du Canada de 1765. Revue

d'histoire de l'Amérique française, 29(3), 323-351.

https://doi.org/10.7202/303462ar d'utilisation que vous pouvez consulter en ligne.

https://apropos.erudit.org/fr/usagers/politique-dutilisation/ 


\section{ÉTUDE CRITIQUE DU RECENSEMENT DU CANADA DE 1765*}

YVES LANDRY

Montréal

Après avoir été nommé premier gouverneur de la «province of Quebec», au lendemain du traité de Paris de 1763, le général James Murray reçut des instructions très détaillées du gouvernement impérial précisant ses nouvelles responsabilités. Deux des quatre-vingtdeux articles de ces instructions prévoyaient la confection d'un recensement de la population et des ressources agricoles. Un autre demandait un état de la population indienne, de son économie et de ses institutions.

72. And You are to report to Us, by Our Commissioners for Trade and Plantations, -

What Quantity of Land is now under actual Improvement and Settlement? What are the chief Articles of Produce and Culture; the annual amount of the Quantity of each; and upon what Terms and Conditions the Inhabitants hold their Lands, either of Cultivation, Rent, or Personal Service?

What is the Quantity, Nature and Property of the Land uncultivated; how much of it is capable of Culture; and what part thereof is private Property?

What is the Number of Inhabitants, Whites and Blacks, distinguishing each? What number of the Former is capable of bearing Arms, and what Number of the Latter is annually necessary to be supply'd in proportion to the Land cultivated?

73. You are from time to time to send unto Us, by Our Commissioners for Trade and Plantations, as foresaid, an Account of

* Cet article fait suite à un travail de recherche en démographie, accompli sous la direction d'Hubert Charbonneau, professeur au département de démographie de l'Université de Montréal. Nous remercions Louise Dechêne et Pierre Tousignant, professeurs au département d'histoire de l'Université de Montréal, pour les précieux conseils qu'ils nous ont prodigués.

RHAF, vol. 29, no 3 (décembre 1975) 
the Increase and Decrease of the Inhabitants, Whites and Blacks, and also an Account of all Persons born, christened and buried.

61. And you are to inform yourself with the greatest Exactness of the Number, Nature and Disposition of the several Bodies or Tribes of Indians, of the manner of their Lives, and the Rules and Constitutions, by which they are governed or regulated ${ }^{1}$.

Le 24 octobre 1765, le secrétaire d'État Henry Seymour Conway invitait le gouverneur Murray à exécuter ces ordres et à fournir «a full and distinct Account of the present State of the said Province ${ }^{2}$. Dès cet automne Murray dut obtempérer à la demande de Conway ${ }^{3}$.

Aucun document ne nous dit comment fut mené ce recensement de 1765. Le gouverneur aurait-il procédé par l'intermédiaire des seigneurs, comme pour les dénombrements du régime français ${ }^{4}$ ? $\mathrm{Com}-$ me les divisions du recensement de 1765 sont paroissiales et non seigneuriales, nous doutons que les autorités aient eu recours aux seigneurs. Murray aurait pu aussi, comme pour les recensements nominatifs du Canada au XVII e siècle ${ }^{5}$, nommer des agents recenseurs qui auraient parcouru toute la province. Mais après le départ des administrateurs français, qui aurait pu à la fois bien connaître l'état de l'œkoumène colonial et jouir de la confiance des habitants? Par élimination, il ne reste plus, à peu près, que les curés. Ceux-ci béné-

\footnotetext{
1 «Instructions to Governor Murray», 7 décembre 1763, publiées par Adam Shortt et Arthur G. Doughty, Documents relating to the Constitutional History of Canada, 1759-1791, second and revised edition (Ottawa, 1918), part 1: 199-203. On aura noté l'ignorance du Colonial Office sur le Canada, croyant qu'il s'agissait là d'une colonie de plantation.

2 Conway à Murray, 24 octobre 1765, Public Record Office, Colonial Office, série 42, vol. 25: 284. Dans sa réponse du 20 août 1766 au nouveau secrétaire d'État Lord Shelburne (Archives Publiques du Canada, MG 21, série B, volume 8: 1), Murray a erronément fait référence à cette lettre de Conway en la datant du 24 octobre 1764 .

3 La mention «dated october 1765» ajoutée au titre qui précède la liste des protestants recensés dans le district de Montréal laisse croire que Murray avait déjà entrepris la confection de ce recensement au moment de la réception de la demande de Conway. "Recensement - Return of the Protestant Inhabitants in the District of Montreal, from his Majesty's Justices of the Peace for the said District dated october 1765», Archives Publiques du Canada (APC), MG 8, G 19, volume 22: 1.

4 Guy Frégault, Le XVIIIe siècle canadien (Montréal, HMH, 1968), 34.

5 Hubert Charbonneau, Yolande Lavoie et Jacques Légaré, «Recensements et registres paroissiaux du Canada durant la période 1665-1668. Étude critique», Population, 25 (1), janvier-février 1970: 116-117.
} 
ficiaient de la sympathie de leurs paroissiens et dépendaient très fortement des bonnes grâces du conquérant ${ }^{6}$.

Quoi qu'il en soit, le secrétaire d'État Conway, par lettre datée du premier avril 1766, manda Murray en Angleterre pour rendre compte de sa conduite et se défendre des accusations de favoritisme envers les Canadiens que les marchands protestants de Québec et de Montréal avaient adressées à Londres. Le gouverneur quitta Québec le 28 juin 1766. C'est une fois arrivé à Londres, le 20 août 1766 , qu'il écrivit une lettre souvent citée à Lord Shelburne, récemment nommé secrétaire d'État. Pour mieux prouver le caractère minoritaire de l'élément britannique dans la province de Québec et, par conséquent, montrer l'impossibilité d'exclure les catholiques du gouvernement, Murray s'appuya sur les résultats du recensement effectué l'année précédente.

\section{STATE OF THE PROVINCE}

It consists of One Hundred and ten Parishes exclusive of the Towns of Quebec and Montreal. These Parishes contain 9722 Houses and 54,575 Christian Souls, they Occupy 955,754 Arpents of Arable Land, in the year 1765 they sowed 180,300 1/2 Minots of Grain, and that year they possessed 12,546 Oxen, 22,724 Cows, 15,039 Young Horned Cattle, 27,064 Sheep, 28,976 Swine, and 13,757 Horses, as appears by the Recapitulation of the Recensement taken by my Order in the year 1765. The Towns of Quebec and Montreal contain about 14,700 Inhabitants. The Savages who are all Roman Catholics, living within the Limits of the Province consist of 7,400 Souls; so the whole exclusive of the Kings Troops doth amount to 76,675 Souls; of which in the Parishes are nineteen Protestant Families; the Rest of that Persuasion, a few half Pay Officers excepted, are Traders, Mecha-

6 Marcel Trudel, «La servitude de l'Église catholique du Canada français sous le régime anglais", Rapport 1963 de la Société historique du Canada: 42-64. Le gouvernement intervint auprès de l'évêque pour que les curés fassent le dénombrement de la population en 1789, 1790, 1822 (deux fois) et en 1825. - L'archiviste Pierre-Georges Roy a lui aussi attribué, mais sans dire pourquoi, aux curés de la province le recensement de 1765: «Le recensement des gouvernements de Montréal et des Trois-Rivières fait par les curés des paroisses, sur l'ordre de leur évêque, pour être soumis aux nouveaux maîtres du pays, nous donne des chiffres révélateurs sur les familles..." (Rapport de l'Archiviste de la province de Québec pour 19361937 (Québec, 1937), lettre au Secrétaire de la province). - Comme le siège épiscopal de Québec était encore vacant en 1764-1765 après le décès de Mgr de Pontbriand en 1760 , c'est sans doute à la suite de lettres circulaires des vicaires généraux du diocèse que le recensement a été entrepris par les curés. 
nics and Publicans, who reside in the two Towns of Quebec and Montreal ${ }^{7}$.

\section{Présentation des sources manuscrites}

La majeure partie des résultats manuscrits du recensement de 1765 se retrouve aujourd'hui aux Archives Nationales du Québec. Ils sont rédigés en français dans un gros cahier de 288 pages, encore en très bon état, aux dimensions de $9^{1} k^{\prime \prime} \times 15^{\prime \prime} \times 3^{\prime \prime} 8$.

Ce cahier fournit des informations pour un ensemble de cent dix paroisses, soit le nombre total de paroisses cité par le gouverneur Murray dans sa lettre du 20 août 1766. Il faut toutefois se montrer prudent: comme le signale le tableau 1, quatre paroisses ont été dédoublées $^{9}$, ce qui réduit le nombre réel de paroisses recensées à 106. Sur le plan géographique, ces paroisses se répartissaient ainsi: 52, soit la moitié, faisaient partie du gouvernement de Québec, 16 de celui de Trois-Rivières et 38 de celui de Montréal.

Poùr 35 paroisses, dont 21 comprises dans le gouvernement de Montréal, le recensement de 1765 peut être qualifié de partiellement nominatif car nous trouvons des renseignements à l'échelle de chaque ménage. Pour les deux autres tiers, concentrés surtout dans le gouvernement de Québec, nous ne possédons que des tableaux récapitulatifs, englobant l'ensemble de la paroisse recensée.

Les recenseurs ont relevé les informations suivantes: d'abord le nom du chef de ménage, et, dans certains cas, sa «qualité ou occupation ${ }^{10}$; suivent le nombre de «maisons» habitées par le ménage, le nombre d'hommes, de femmes, d' «enfants mâles au-dessus de 15 ans », d' "enfants mâles au-dessous de 15 ans », d' "enfants femelles», de "domestiques mâles au-dessus de 15 ans", de «domestiques mâles au-dessous de 15 ans», de «domestiques femelles» et d'étran-

7 Murray à Shelburne, 20 août 1766, APC, MG 21, série B, volume 8: 1.

8 Archives Nationales du Québec (ANQ), QBC-8-1. Le contenu de ce cahier a été reproduit par Pierre-Georges Roy, op. cit.: 1-121. Nous discuterons plus loin (p. 350) de la valeur de cette transcription.

9 En 1765, le Petit St-Ours et le Grand St-Ours faisaient partie d'une seule et même paroisse appelée St-Ours; la «R[iviè]re St-Charles depen[dan]t de Charlebourg» était partie intégrante de la paroisse Charlesbourg; Berthier (en Haut) et la Petite Rivière de Berthier formaient Berthierville; «D'autré» et «Nauray» constituaient Lanoraie.

10 La «qualité ou occupation» du chef de ménage n'a pas été signalée d'une façon systématique. Seules les professions de certains notables (curé, seigneur, bailli...) ont été inscrites sous cette colonne. 
gers; viennent enfin les données sur l'agriculture: le nombre d'arpents de terre, le nombre de minots en «semence en 1765 », le nombre de bœufs, de vaches, de "taurailles", de moutons, de chevaux et de cochons.

Les Archives Publiques du Canada ne possèdent des manuscrits du recensement de 1765 que pour sept paroisses concentrées dans le gouvernement de Trois-Rivières: Cap-de-la-Madeleine, Champlain, Batiscan («La Grande Côte de Batiscan» et «La Rivière Batiscan»), Ste-Anne-de-la-Pérade, St-Pierre-les-Becquets, Gentilly et Bécancour ${ }^{11}$. Sur le plan de la présentation, les données de ces sept paroisses occupent, mis à part le feuillet de récapitulation, treize feuilles manuscrites non reliées et pliées séparément.

À première vue, l'allure de ces feuillets diffère de celle du cahier conservé aux Archives Nationales du Québec. D'abord l'écriture est différente. Puis les tableaux ont été rédigés d'une façon visiblement plus soignée. Si nous poussons l'observation, nous constatons rapidement que ces feuillets sont plus complets. En effet, la «qualité ou occupation" de chaque chef de ménage est ici indiquée systématiquement, tandis qu'aux Archives Nationales elle n'était écrite que très occasionnellement. Les différentes catégories de la population (hommes, femmes, enfants mâles au-dessus de 15 ans, au-dessous de 15 ans, etc.) sont toutefois les mêmes. Mais si nous regardons plus loin du côté des données agricoles, nous remarquons les nouveautés suivantes : on a fait la distinction entre les arpents de terre «désertés » et ceux «en bois de bout», alors qu'aux Archives Nationales on n'en avait que le total; puis on possède la quantité des semences en 1765 en «bled», en «poids» et en «avoine», tandis qu'encore une fois le cahier des Archives Nationales n'en fournissait que le total.

\section{Critique des sources manuscrites}

Après cette brève description des documents conservés aux Archives Nationales du Québec et aux Archives Publiques du Canada, nous établirons notre critique en tentant de répondre aux trois questions suivantes: $1^{\circ}$ Toutes les paroisses existant dans la province de

11 APC, cote RG4, Al, vol. S-11, feuillets 4496 à 4521 . On peut également consulter aux Archives Publiques du Canada la liste des protestants du district de Montréal (MG 21, série B, volume 8: 96-100). 


\section{TABLEAU 1}

Paroisses recensées et/ou existantes en 1765, d'après la date d'ouv'erture des registres paroissiaux,

le gouvernement et le type de manuscrit conservé

\section{Paroisses}

Longue-Pointe

Lachine

St-Laurent

Pointe-Claire

Ste-Geneviève-de-

Pierrefonds

Vaudreuil

Ste-Anne-de-Bellevue

Ile Perrot

Les Cèdres

Verchères

Boucherville

Sorel

Chambly

St-Mathias

St-Antoine-sur-Richelieu

St-Charles-sur-Richelieu

St-Denis-sur-Richelieu

Contrecoeur

Petit St-Ours

Longueuil

Varennes

Cap-de-la-Madeleine

Champlain

Batiscan

Ste-Anne-de-la-Pérade

St-Pierre-les-Becquets

Gentilly

Bécancour

Baie-du-Febvre

St-François-du-Lac

Nicolet

\section{Date d'ouverture des registres} paroissiaux $^{1}$
Gouvernements de Trois-Rivières
Manuscrit conservé oui non

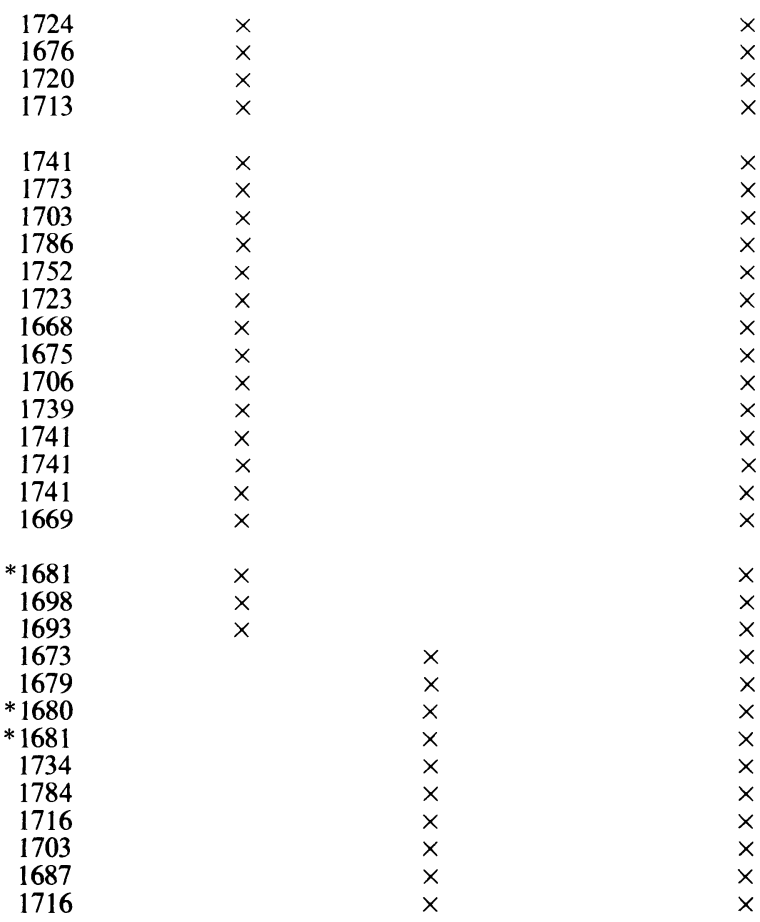


Yamaska

Baie des Chaleurs,

Ristigouche

1813

Kamouraska

Rivière-Ouelle

Ste-Anne-de-la-

St-Roch-des-Aulnaies 1734

St-Jean-Port-Joli 1767

L'Islet 1679

Cap-St-Ignace $\quad 1679$

Montmagny 1679

St-Pierre-du-Sud $\quad 1727$

St-François

Berthier

St-Vallier

St-Michel

St-Charles

1713

1693

1749

Beaumont

Ste-Marie-de-Beauce

St-Joseph-de-Beauce $\quad 1738$

St-Henri 1766

Lauzon

1679

St-Nicolas

1694

St-Antoine-de-Tilly $\quad 1702$

Ste-Croix

1727

Lotbinière 1692

St-Jean-Deschaillons $\quad 1741$

Grondines

1680

Deschambault

713

Cap-Sante

679

Les Écureuils 1742

Neuville

1679

1693

L'Ancienne-Lorette 1676

Ste-Foy $\quad 1679$




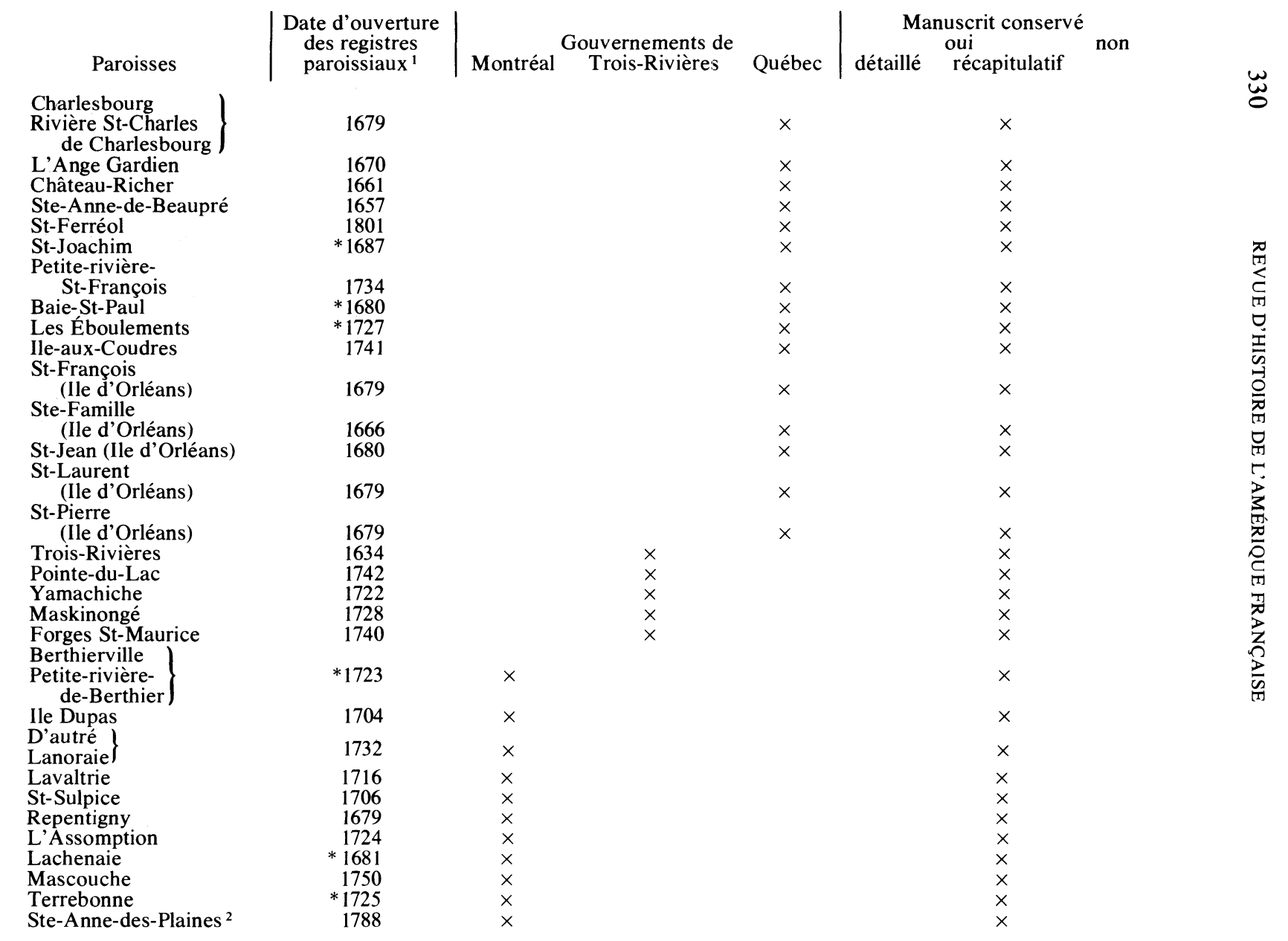


St-François-de-Sales

(Ile Jésus)

St-Vincent-de-Paul

Rivière-des-Prairies

Sault-au-Récollet

Pointe-aux-Trembles

Québec

Postes du Domaine du

Roi (Tadoussac et

$$
\text { autres lieux) }
$$

Rimouski

1668

Trois-Pistoles

Loretteville

1701

Leauceville

1713

1761

Louiseville

1765
1714

Ste-Geneviève-de-

$$
\text { Batiscan }
$$

* 1727

Montréal

Laprairie

Oka

1642

1642
1670

Caughnawaga

Châteauguay

St-Constant

St-Philippe

St-Régis

St-Cuthbert

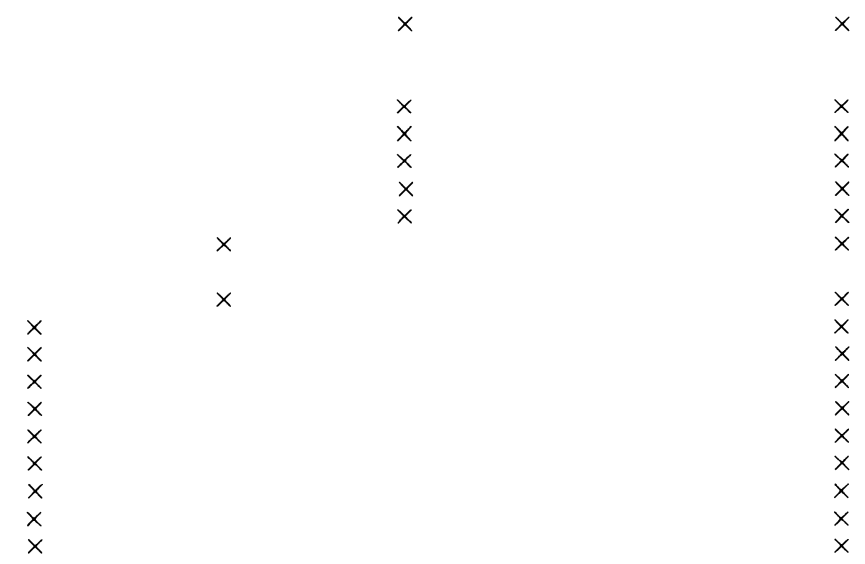

$\times$

1 D'après les estimations du Programme de recherche en démographie historique du département de démographie de l'Université de Montréal. Ces dates indiquent, pour chaque paroisse, l'année du plus ancien registre conservé dans les archives paroissiales ou civiles (dans ce dernier cas, les dates sont marquées d'un astérisque).

2 Nous avons assimilé, sous toutes réserves, «Mascouche de Terrebonne» à la future paroisse de Ste-Annedes-Plaines.

${ }^{3}$ Les registres du Lac des Deux-Montagnes (Oka) de 1721 à 1922 ont été détruits dans l'incendie du presbytère d'Oka en 1922. 
Québec en 1765 ont-elles été recensées? $2^{\circ}$ Sur un plan purement arithmétique, les additions des données chiffrées ont-elles été effectuées avec exactitude par le compilateur? $3^{\circ}$ Toute la population catholique de la province de Québec a-t-elle été dénombrée?

Plusieurs historiens, à la suite du Bureau de la Statistique du Canada qui l'avait déjà signalé ${ }^{12}$, ont noté que les villes de Québec et de Montréal n'avaient pas été recensées. Un seul cependant, Gustave Lanctôt ${ }^{13}$, a semblé s'apercevoir que certaines paroisses rurales avaient aussi été oubliées.

L'ouverture de registres dans une paroisse constitue un événement qui peut témoigner du degré d'importance de la population qui y vit. Connaissant cette date avec précision pour toutes les paroisses fondées avant $1875^{14}$, nous avons pu dresser la liste des paroisses existantes en 1765 et qui étaient susceptibles d'être recensées. Le résultat de cette opération apparaît au tableau 1. Parmi dix-sept paroisses existantes en 1765 mais non recensées dont les noms apparaissent en fin de liste, il faut d'abord distinguer toutes celles qui semblent avoir été volontairement omises du recensement, soit les paroisses urbaines de Notre-Dame-de-Québec et de Notre-Dame-deMontréal et les missions indiennes ${ }^{15}$ : Postes du Domaine du Roi (Tadoussac et autres lieux), Lorette (Loretteville), Lac des DeuxMontagnes (Oka), Sault St-Louis (Caughnawaga) et St-Régis ${ }^{16}$. Après cette première élimination, il reste donc dix paroisses, rurales et habitées de blancs, qui auraient dû apparaître dans la liste des paroisses recensées et qui n'y figurent pas: Rimouski, Trois-Pistoles, St-François-de-Beauce (Beauceville), Rivière-du-Loup-en-Haut (Louiseville), Ste-Geneviève-de-Batiscan, Laprairie, Châteauguay, St-Constant, St-Philippe et St-Cuthbert.

\footnotetext{
12 Canada, Recensement du Canada - 1870-71, volume IV: Recensements du Canada. 1665 à 1871 (Ottawa, 1876) : 68. 342 .

13 Gustave Lanctôt, Histoire du Canada (Montréal, Beauchemin, 1964), 3 :

14 Nous remercions le Programme de recherche en démographie historique du département de démographie de l'Université de Montréal, de nous avoir communiqué ces données.

15 À l'éxception des «Sauvages de Resticouche» dont l'énumération occupe une place à part à la fin du cahier conservé aux Archives Nationales du Québec.

16 Le village des Abénakis sur la rivière St-François, dont la fondation remonte à la fin du XVIIe siècle, n'a manifestement pas été intégré à la paroisse voisine de St-François-du-Lac: aucun nom d'apparence indigène ne figure dans la liste des habitants recensés.
} 
Nous devons cependant préciser que les populations de certaines de ces paroisses, surtout les plus récentes, ont pu être recensées avec celles de paroisses voisines ou desservantes. Le cas le plus patent est celui de la paroisse St-François-de-Beauce, dont les registres contiennent quelques actes de sépulture de 1765 mais qui a continué à être desservie par la paroisse St-Joseph-de-Beauce jusqu'en $1783^{17}$.

Notons, en dernier lieu, que le phénomène contraire s'est aussi produit: dix «paroisses» ont été recensées avant que ne s'ouvrent leurs registres. Il s'agit de St-Henri, St-Jean-Port-Joli, Vaudreuil, Gentilly, Ile Perrot, Ste-Anne-des-Plaines, Bonaventure, St-Ferréol, Rivière-du-Loup (en Bas) et Gaspé. D'autre part, deux «paroisses » ont été recensées après que leurs registres eurent été fermés: Ristigouche (clôture en 1761) et les Forges du St-Maurice (en 1762). Ces faits illustrent bien l'écart temporel entre la réalité du peuplement et la tenue de registres paroissiaux et laissent croire à l'existence possible d'autres populations marginales qui auraient échappé au recensement.

Une des conditions que doit respecter tout document, en particulier le document quantitatif, pour assurer sa crédibilité, est la cohérence. Afin de mesurer le degré de cohérence du recensement de 1765 , nous avons vérifié toutes les additions (à l'exception de celles concernant les informations agricoles) effectuées dans le cahier conservé aux Archives Nationales du Québec.

Les données contenues dans ce cahier nous ont permis de mettre au point deux séries de nombres totalisant la population de chacune des paroisses recensées au Canada en 1765 (tableau 2). La première colonne donne les nombres bruts qui constituent, au niveau de chaque paroisse, la somme horizontale des totaux de chaque catégorie (hommes, femmes, enfants mâles, etc.) inscrits par le compilateur. Cette série ferme donc les yeux sur d'éventuelles erreurs d'addition commises à l'endroit des totaux de chaque catégorie. La seule correction que nous nous sommes permise concerne le total de ces nombres bruts: nous avons porté 54.298 habitants au lieu des 54.275

17 Raoul Raymond, «Relevé sommaire des paroisses fondées avant 1800 », Mémoires de la Société généalogique canadienne-française, IX, 3 et 4 (juillet et octobre 1958): 149. 
TABLEAU 2

Population totale des paroisses recensées en 1765, selon différentes sources

Paroisses

Longue-Pointe

Lachine

St-Laurent

Pointe-Claire

Ste-Geneviève-de-

Pierrefonds

Vaudreuil

Ste-Anne-de-Bellevue

Ile Perrot

Les Cèdres

Verchères

Boucherville

Sorel

Chambly

St-Mathias

St-Antoine-sur-Richelieu

St-Charles-sur-Richelieu

St-Denis-sur-Richelieu

Contrecoeur

Petit St-Ours

Grand St-Ours

Longueuil

Varennes

Cap-de-la-Madeleine

Champlain

Batiscan

Ste-Anne-de-la-Pérade

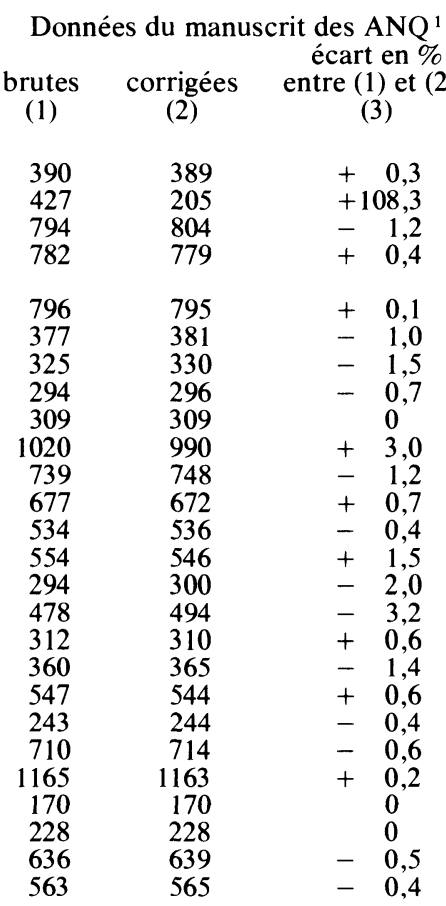

Données publiées

$$
\text { dans le }
$$

Rec. du Canada 1870-71

(4)

390

423
795

795

796
377

377
325

294

309

963

748
1009

555

309

478

312

551

243

243
714

1168

170
228

228

636
563
Écart en $\%$ entre (1)
et $(4)$
$(5)$
Ecar
en $\%$
entre (2)
(6)

$0+0,3$

\begin{tabular}{ll}
$-0,9+106,3$ \\
\hline
\end{tabular}

$0,1-1,1$
$+0,1$
$+\quad 0,5$

$0+0,1$

$+\quad 0,1$
$-\quad 1,0$

$-1,0$
$-\quad 1,5$

- 0,7

0
$-\quad 2,7$

2,7
$-\quad 0$

$+50,1$

$+\quad 1,5$

$+\quad 1,6$

$+\quad 3,0$

- 3,2

$+\quad 0,6$

$+\quad 1,6$

$+1,3$

+
$-\quad 0,4$

$\begin{array}{ll}+0,6 & \\ +0,3 & 0 \\ & 0,4\end{array}$

$\begin{array}{ll}\mathbf{0} & \mathbf{0} \\ 0 & 0\end{array}$

0,5
$-\quad 0,4$

0,5
$-\quad 0,4$ 
St-Pierre-les-Becquets Gentilly

Bécancour

Baie-du-Febvre

St-François-du-Lac

Nicolet

Yamaska

Baie des Chaleurs,

Bonaventure

Gaspé

Ristigouche

Rivière-du-Loup

Kamouraska

Rivière-Ouelle

Ste-Anne-de-la-Pocatière

St-Roch-des-Aulnaies

St-Jean-Port-Joli

L'Islet

Cap-St-Ignace

Montmagny

St-Pierre-du-Sud

St-François

Berthier

St-Vallier

St-Michel

St-Charles

Beaumont

Ste-Marie-de-Beauce

St-Joseph-de-Beauce

St-Henri

Lauzon

St-Nicolas

St-Antoine-de-Tilly

Ste-Croix

Lotbinière

St-Jean-Deschaillons

Grondines

Deschambault

Cap-Santé

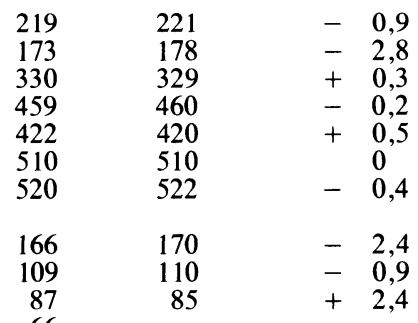

871

810

608
555

392

599

598

1094

612

878

960
385

364

499

288

794

427

553

388

184

254
428

818

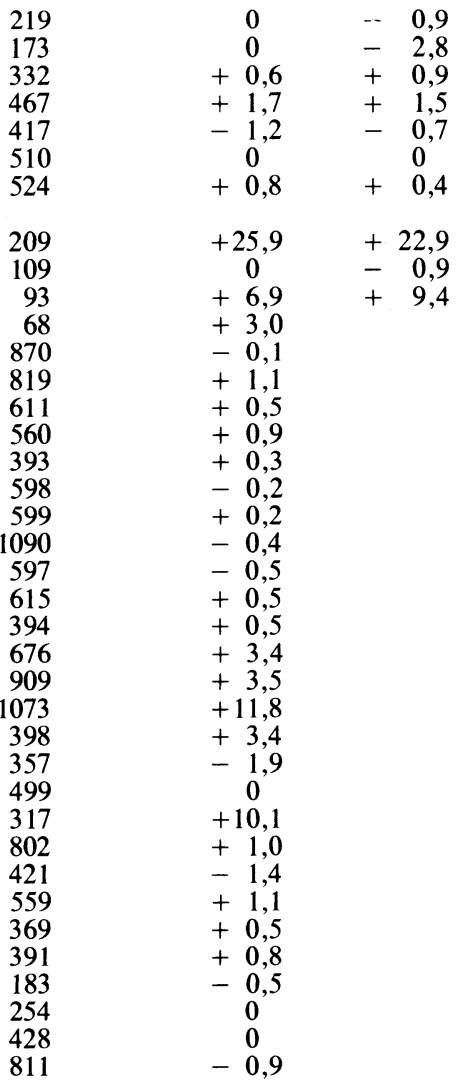


Paroisses

Les Écureuils

Neuville

St-Augustin

L'Ancienne-Lorette

Ste-Foy

Beauport

Charlesbourg

Rivière St-Charles de Charlesbourg

L'Ange Gardien

Château-Richer

Ste-Anne-de-Beaupré

St-Ferréol

St-Joachim

Petite-rivière-St-François

Baie-St-Paul

Les Éboulements

Ile-aux-Coudres

St-François (Ile d'Orléans)

Ste-Famille (Ile d'Orléans)

St-Jean (Ile d'Orléans)

St-Laurent (Ile d'Orléans)

St-Pierre (Ile d'Orléans)

Trois-Rivières

Pointe-du-lac

Yamachiche

Maskinongé

Forges St-Maurice

Berthierville

Petite-rivière-de-Berthier

Ile Dupas

D'autré

Lanoraie

$$
\begin{array}{r}
\text { Données du manuscrit des ANQ }{ }^{1} \\
\text { écart en \% }
\end{array}
$$
brutes corrigées

(2)

(3)

303

704

642

1049

350

899

1263

338

415

304

360

124

364

153

535

152

211

459

523

471

468

644

181

636

352

273

635

372

280

183

\author{
Rec
} dans le
du Canada $1870-71^{3}$

(4)
(8)-71

en $\%$

entre (1)

et (4)

Écart

en $\%$

entre (2)

(5)

(6)

$\begin{array}{r}0,7 \\ \hline\end{array}$

$+0,7$
$-\quad 0,6$
$+23,8$

$+23,8$

$-\quad 9,7$
$+\quad 3,4$

3,4
$+\quad 0,9$

$-0,9$
$-\quad 1,9$

$-12,1$

12,1
$+\quad 0,7$

$+\quad 0,7$
$+62,8$

$+0,6$

+
$+\quad 0,8$

$-0,5$

$-0,5$
$-0,7$

0,9
$+\quad 2,0$

$+2,0$
-

$+0,9$

$-0,5$

$-0,4$

$+0,2$

$+0,4$

$+0,6$

0

$+0,6$

0

0,3
0

2,2
0

0

$\mathbf{0}$
$\mathbf{0}$ 


\begin{tabular}{lrrr} 
Lavaltrie & 327 & 327 & 0 \\
St-Sulpice & 567 & 567 & 0 \\
Repentigny & 712 & 712 & 0 \\
L'Assomption & 1000 & 1000 & 0 \\
Lachenaie & 353 & 352 & 0,3 \\
Mascouche & 542 & 542 & 0 \\
Terrebonne & 540 & 540 & 0 \\
Ste-Anne-des-Plaines & 436 & 436 & 0 \\
Ste-Rose & 835 & 835 & 0 \\
St-François-de-Sales & & & 0,4 \\
$\quad$ (Ile Jésus) & 234 & 233 & 0 \\
St-Vincent-de-Paul & 1311 & 1311 & 0 \\
Rivière-des-Prairies & 366 & $366^{6}$ & 0,4 \\
Sault-au-Récollet & 258 & 257 & 0 \\
Pointe-aux-Trembles & 459 & 459 & 1,5 \\
\multicolumn{1}{c}{ Total } & 54.298 & 55.110 &
\end{tabular}

1 Les données brutes proviennent du cahier conservé aux Archives Nationales du Québec, cote QBC-8-1; elles ont été publiées par P.-G. Roy, Rapport de l'Archiviste de la province de Québec pour 1936-1937 (Québec, 1937 ): 1-121. Les données corrigées sont de nous; elles n'effacent que les erreurs d'addition des données brutes et que pour les seules paroisses dont on possède le recensement partiellement nominatif.

2 (1) - (2)

$(2)$

${ }^{3}$ Canada, Recensement du Canada - 1870-7I, volume IV: Recensements du Canada. 1665 à 187I (Ottawa, 1876) : 64-65.

$$
\begin{aligned}
& \frac{4(4)-(1)}{(1)} \\
& 5 \frac{(4)-(2)}{(2)}
\end{aligned}
$$

6 On lit ibid. le nombre 360, mais il s'agit d'une erreur d'addition (la seule, d'ailleurs, dans cette version du recensement). 
marqués erronément par le compilateur. La deuxième colonne donne les nombres corrigés que nous n'avons pu établir, forcément, que pour les paroisses pour lesquelles nous possédions des données au niveau de chaque ménage. Nous avons ici fait les corrections d'addition qui s'avéraient nécessaires chaque fois que nous relevions, pour chaque paroisse, des erreurs dans les totaux des diverses catégories, pour ensuite effectuer une nouvelle somme de ces totaux rectifiés.

Les résultats de la comparaison qu'établit le tableau 2 entre nombres bruts et nombres corrigés sont très révélateurs: sur trentesix paroisses, seulement quatre ont obtenu deux nombres identiques, et encore faut-il préciser que deux de ces quatre paroisses n'ont réussi ce «tour de force» que grâce à l'effet compensateur de leurs erreurs d'addition. C'est donc dire que trente-quatre des trente-six paroisses dont nous possédions le recensement partiellement nominatif ont compté au moins une erreur d'addition dans leurs totaux des diverses catégories.

Ces erreurs ont-elles été commises volontairement? Pour treize des trente-deux paroisses dont l'effectif total a été affecté par ces erreurs, leur population s'est accrue, tandis que pour les dix-neuf autres elle a diminué. Si l'on excepte la paroisse de Lachine, pour laquelle l'écart entre nombres brut et corrigé est considérable (son effectif total a été augmenté de $108 \%$ ), les différences au niveau des totaux des catégories ont eu tendance à se compenser, de telle sorte que l'effectif brut de chaque paroisse n'a différé, règle générale, de l'effectif corrigé que de quelques unités et que la population totale de ces trente-deux paroisses n'a été réduite que de 1,6\%.

Si ces variations sont trop discordantes et trop faibles pour avoir été conçues dans un but intentionné et être statistiquement significatives, les erreurs d'addition presque systématiques qui sont à leur origine, si compensatrices soient-elles, ébranlent sérieusement notre confiance en la validité des nombres cités dans ce document. Voyons comment ces erreurs ont été commises.

Dans ces paroisses où les résultats partiellement nominatifs du recensement nous sont parvenus, le compilateur procédait ainsi. À la première page, il énumérait, en colonnes, les noms des premiers chefs de ménage recensés et les caractéristiques quantitatives de chacun de ces ménages. Puis, en tête de la deuxième page, il écrivait: "De l'autre Part» et additionnait chacune des colonnes de la page précédente; les lignes suivantes, il poursuivait le recensement des autres ménages. À la troisième page et les autres, il effectuait un sous-total 
cumulatif des colonnes des pages précédentes. À la dernière page, après avoir calculé le dernier sous-total cumulatif, il énumérait les derniers ménages et leurs caractéristiques et établissait le total en additionnant au dernier sous-total cumulatif les données de cette dernière page. C'est à l'occasion de ce dernier calcul, à la toute fin du décompte de chaque paroisse, qu'ont été commises presque toutes les erreurs. Il ne s'agissait pourtant, le plus souvent, que de très simples additions, alors que les calculs précédents avaient parfois été plus difficiles et pourtant effectués sans erreur ${ }^{18}$.

Sans avoir été commises dans le but intentionné de grossir ou de diminuer l'effectif total des populations (sauf peut-être dans le cas de la paroisse de Lachine), les fréquentes erreurs d'addition relevées dans ce document laissent peu de doute quant à la négligence de son compilateur. Au surplus, le fait que ces erreurs n'aient pas été normalement disséminées tout au long du processus d'addition mais surtout concentrées à la fin, laisse à penser qu'il y a eu manipulation des résultats, sans graves conséquences pour les résultats globaux (vu la compensation des erreurs) mais de nature à rendre suspecte toute utilisation de résultats fragmentaires.

L'examen des treize feuillets manuscrits conservés aux Archives Publiques du Canada confirme la responsabilité de l'auteur de la compilation conservée aux Archives Nationales dans l'altération de ses résultats. Nous avons vérifié toutes les additions effectuées dans ces feuillets: aucune erreur. Le tableau 3 montre que l'effectif total de la population de chacune des sept paroisses considérées se rapproche beaucoup plus de celui que nous avons calculé à partir du cahier des Archives Nationales que de celui de sa version brute. Cette constatation, jointe à la subdivision des catégories déjà notée, nous permettent de croire que ces quelques feuilles, qu'on a réussi à sauver, constitueraient un spécimen de documents qui auraient servi à la rédaction du cahier des Archives Nationales. Peut-être s'agit-il des rôles mêmes du recenseur chargé de ces sept paroisses. On peut supposer que, par la suite, le copiste aurait négligé de transcrire les occupations des chefs de ménage les plus courantes (e.g. laboureur), de même que les sous-catégories des «arpents de terre» et des «semences en 1765 ». Le recouvrement de tous les feuillets qui ont servi à cette transcription contribuerait certainement à ré-

\footnotetext{
18 Roy, op. cit.: 5-6, en reproduisant tous les sous-totaux de la paroisse de Lachine, a mis en évidence les incohérences de la compilation.
} 


\section{TABLEAU 3}

Population totale de sept paroisses du gouvernement de Trois-Rivières, selon les trois sources du recensement de 1765

\begin{tabular}{lcccc} 
& $\begin{array}{c}\text { Données } \\
\text { du }\end{array}$ & $\begin{array}{c}\text { Données du manuscrit } \\
\text { manuscrit } \\
\text { des APC }\end{array}$ & $\begin{array}{c}\text { des ANQ } \\
\text { brutes }\end{array}$ & $\begin{array}{c}\text { Données publiées } \\
\text { dans le }\end{array}$ \\
corrigées & $\begin{array}{c}\text { Rec. du Canada } \\
\text { l870-7 }\end{array}$ \\
Cap-de-la-Madeleine & 170 & 170 & 170 & 170 \\
Champlain & 228 & 228 & 228 & 228 \\
Batiscan & 642 & 636 & 639 & 636 \\
Ste-Anne-de-la-Pérade & 570 & 563 & 565 & 563 \\
St-Pierre-les-Becquets & 221 & 219 & 221 & 219 \\
Gentilly & 178 & 173 & 178 & 173 \\
Bécancour & 329 & 330 & 329 & 332 \\
\multicolumn{1}{c}{ Total } & 2338 & 2319 & 2330 & 2321
\end{tabular}

1 Archives Publiques du Canada, cote RG4, Al, vol. S-11: \#4496 à 4521.

2 Les données brutes proviennent des Archives Nationales du Québec, cote QBC-8-1; elles ont été publiées par P.-G. Roy, Rapport de l'Archiviste de la province de Québec pour 1936-1937 (Québec, 1937): 1-121. Les données corrigées sont de nous; elles n'effacent que les erreurs d'addition des données brutes.

3 Canada, Recensement du Canada - 1870-71, volume IV: Recensements du Canada. 1665 à 1871 (Ottawa, 1876) : 64-65.

soudre les contradictions entre les résultats des différentes versions du recensement ${ }^{19}$.

L'évaluation du sous-dénombrement par lequel pèche un recensement est une opération très délicate puisqu'elle suppose que l'on possède d'autres sources démographiques plus sûres que celles qu'on veut critiquer. Comme ces sources auxiliaires ne sont jamais parfaites, de même ces évaluations du sous-dénombrement sont-elles toujours relatives.

\footnotetext{
19 Il n'est pas certain que les autres feuillets aient été détruits. Nous avons lu sur la première page de garde du cahier conservé aux Archives Nationales du Québec, écrit vraisemblablement de la main d'un archiviste: "I am informed that Two more Vols. are in the Department of Archives - Ottawa [initiales] 28/3, 1925 " Nous n'avons malheureusement rien trouvé de tel aux Archives Publiques du Canada.
} 
Rares sont les historiens qui se sont attaqués jusqu'ici au problème du sous-dénombrement posé par les recensements. Georges Langlois, le premier véritable historien démographe au Québec, est l'auteur qui a fait les calculs les plus élaborés au sujet du recensement de 1765 , sans toutefois être aussi critique que nous l'aurions espéré. Il a abordé les résultats du recensement de $1765^{20}$ lorsqu'il a cherché à vérifier si l'évaluation traditionnellement acceptée de la population canadienne en 1763 (65,000 habitants) était exacte. Pour ce faire, il a utilisé les données du recensement de 1754 et les statistiques du mouvement naturel de la population telles qu'établies par Cyprien Tanguay ${ }^{21}$ :

On admet généralement que le traité de Paris céda à la GrandeBretagne soixante-cinq mille Canadiens. Avant d'aller plus loin, il faut bien établir ce nombre.

Nous avons deux repères certains ou que nous pouvons accepter comme tels: le résultat des recensements de 1754 et de 1765 : 55,009 et 68,810 [sic], respectivement. Si nous ajoutons au recensement de 1754 les excédents annuels [les naissances moins les décès], nous obtenons la somme suivante:

\begin{tabular}{|c|c|c|}
\hline \multicolumn{2}{|c|}{ Population en 1754} & 55,009 \\
\hline \multicolumn{2}{|c|}{ Excédent en 1755} & 999 \\
\hline , &,$\quad 1756$ & 1,061 \\
\hline , & , 1757 & 1,123 \\
\hline ," & , 1758 & 540 \\
\hline , & , 1759 & 930 \\
\hline , & ,' 1760 & 886 \\
\hline , & , 1761 & 1,433 \\
\hline , & , 1762 & 2,123 \\
\hline \multicolumn{2}{|c|}{ Population en 1763} & 64,104 \\
\hline
\end{tabular}

Il convient d'ajouter à ce total une partie de l'excédent de 1754 pour comprendre dans la population les enfants nés en 1754 dans les mois qui ont suivi le recensement; cet excédent était de 1,696. Si l'on en ajoute la moitié, on obtient:

$$
\begin{array}{r}
64,104 \\
848 \\
\hline 64,952
\end{array}
$$

20 Langlois s'est servi des résultats publiés par le Bureau de la Statistique du Canada (Canada, Recensement du Canada - 1870-71, volume IV: Recensements du Canada, 1665 à 1871 (Ottawa, 1876): 65). Nous étudierons cette version imprimée du recensement de 1765 , infra, p. 346.

21 Ibid.: 61, et volume V: Recensement dı Canada. 1608 à 1876 (Ottawá, 1878): 204-207. 
Il est déjà acceptable d'arrondir à 65,000 et l'on peut dire qu'à la date du 10 février, les naissances survenues en 1763 contribuent à justifier ce faible coup de pouce.

On n'a qu'à continuer ce calcul jusqu'en 1765 pour en arriver au résultat du recensement de cette année:

\begin{tabular}{llr} 
Population à la fin de & 1762 & 64,104 \\
Excédent en & 1763 & 2,626 \\
Excédent en & 1764 & 2,605 \\
\hline Population au début de 1765 & 69,335
\end{tabular}

La marge de près de cinq cents entre ce nombre et celui du recensement est comblé de la même façon que précédemment par une partie de l'excédent de $1765^{22}$.

Si l'on se fie à cette démonstration qui a le mérite de combiner le mouvement naturel à l'état de la population, le recensement de 1765 aurait fourni une juste évaluation de la population de la province de Québec. La logique du raisonnement de Langlois repose toutefois sur deux postulats qui peuvent être facilement contestés. Le premier est l'exactitude du recensement de 1754. Un bref coup d'œil sur les résultats de ce recensement ${ }^{23}$ n'a pas de quoi nous rassurer. Plusieurs des chiffres ont manifestement été arrondis ${ }^{24}$, ce qui laisse croire qu'ils sont plus le fruit d'une évaluation grossière (d'où les risques élevés d'erreurs) que d'un véritable recensement.

Le second postulat au raisonnement de Langlois est l'absence de migrations, ou encore l'équilibre entre l'émigration et l'immigration. Malheureusement, les données sont loin d'être claires à ce sujet. Langlois soutient que,

de la population civile, il n'y eut guère que certaines classes de la population à émigrer; d'autre part, comme au moins cinq cents soldats se firent licensier [sic] au Canada et s'y fixèrent ${ }^{25}$ on peut supposer que leur nombre compensa celui des émigrés et que le total de la population civile ne fut pas affecté par l'exode des citadins ${ }^{26}$.

22 Georges Langlois, Histoire de la population canadienne-française (première édition, Montréal, Albert Lévesque, 1934), 157-159.

23 Canada, Recensement du Canada - 1870-71, volume IV : Recensements du Canada. 1665 à 1871 (Ottawa, 1876): 61.

24 Par exemple, les chefs de famille auraient été au nombre de 5.000 dans les paroisses rurales et de 1.200 à Québec, 20 à Trois-Rivières et 600 à Montréal.

${ }_{25}$ Émile Salone, La colonisation de la Nouvelle-France (Paris, Guilmoto, $1905), 447$.

26 Langlois, op. cit., 162. Le démographe Jean-Noël Biraben a repris cette thèse dans son article "Le peuplement du Canada français", Annales de démographie historique - 1966: 125 . 
Cette vue est contestée par Michel Brunet qui rappelle que «des études récentes établissent qu'au moins deux mille Canadiens quittèrent leur pays natal ${ }^{27}$ ». Notre intention n'est pas de trancher ici le débat mais de simplement souligner que le second postulat de Langlois est contestable et qu'il y a là matière à recherche ${ }^{28}$.

Plus près de nous, les démographes Jacques Henripin et Yves Péron se sont aussi penchés sur les résultats du recensement de 1765 . Ils ont en effet cherché à vérifier la validité des recensements de 1765,1784 et 1790 en comparant leurs données avec l'excédent cumulé des naissances sur les décès depuis 1608. Ils ont conclu que ces recensements avaient sous-dénombré la population catholique du Québec.

Il est possible de comparer les évaluations reposant sur les résultats des recensements à l'excédent cumulé des naissances sur les décès depuis 1608 tel que calculé par Tanguay. La concordance entre les deux séries est bonne pour les périodes 1710-1760 et 1830-1860. Les désaccords du début et de la fin de notre période s'expliquent aisément: rôle de l'immigration avant 1710 , de l'émigration après 1860 . [...] Les désaccords entre 1760 et 1830 n'ont pas la même origine: comme on admet généralement que les migrations de catholiques ont été faibles au début du régime anglais, ces écarts ne peuvent se comprendre sans un important sous-dénombrement en 1765,1784 et $1790^{29}$.

À partir de l'excédent naturel cumulé depuis 1608 , les auteurs ont calculé des populations moyennes par période décennale; pour les années 1761-1770 (donc pour la fin de l'année 1765), ils ont établi l'effectif moyen des habitants catholiques à 80.300.

Cette nouvelle estimation de la population canadienne en 1765 n'est pas à l'abri de toute critique. D'abord, elle repose essentiellement sur les statistiques du mouvement de la population établies au siècle dernier par Tanguay. Henripin et Péron ont reconnu les

27 Michel Brunet, La présence anglaise et les Canadiens (Montréal, Beauchemin, 1958), 57 note 14; Les Canadiens après la conquête, 1759-1775 (Montréal, Fides, 1969), 74.

28 L'évaluation du nombre d'immigrants acadiens au Canada entre 1756 et 1763 est l'une des voies à explorer à ce sujet. Le démographe Raymond Roy ( $L a$ croissance démographique en Acadie de 1671 à 1763, mémoire de maîtrise en démographie, Université de Montréal, avril 1975: 85-86) a récemment avancé le chiffre de trois mille.

29 Jacques Henripin et Yves Péron, «La transition démographique de la province de Québec", La population du Québec: études rétrospectives, par Hubert Charbonneau (s.1., Boréal Express, 1973), 26-27. 
«nombreuses faiblesses» de cette œuvre mais comme le Programme de recherche en démographie historique de l'Université de Montréal n'a pas encore revisé ces statistiques pour le milieu du XVIIIe siècle, ils ont dû s'en contenter et espérer qu'elles n'étaient pas trop éloignées de la réalité. La seconde critique vient de Langlois et concerne le procédé utilisé par les deux démographes.

Il semble, à première vue, qu'en soustrayant du total accumulé des naissances le total accumulé des décès on obtienne, au total accumulé des excédents, le chiffre de la population à une date donnée. [...] Ce procédé est trompeur, car il traîne avec lui une erreur systématique qui reste insignifiante et négligeable si le calcul porte sur une période très courte, mais qui devient assez importante pour vicier le résultat si le calcul s'applique à une longue période. En effet, le nombre des décès enregistrés comprend le décès d'au moins dix mille personnes dont la naissance a été enregistrée en France: les dix mille colons passés au Canada; par contre, le total des naissances comprend la naissance d'un nombre indéterminé de personnes qui sont allées mourir dañs des endroits tels que leur décès n’a pas été enregistré au Canada: les colons rentrés en France après un séjour plus ou moins prolongé dans la colonie, les miliciens morts à la guerre et dont le décès n'a pas été enregistré, les trappeurs et chasseurs morts dans les bois et portés disparus sans que leur décès ait été constaté, les coureurs de bois passés chez les Anglais ou en Louisiane, les aventuriers embauchés par des corsaires ou établis dans les «Pays d'en Haut». Deux facteurs d'erreur dont l'influence s'exerce, il est vrai, en sens inverse et qui pourraient tendre à se compenser: mais cette compensation même est incertaine et rend le total accumulé des excédents depuis 1608 trompeur et inutilisable ${ }^{30}$.

En dépit de cette sévère mise en garde de Langlois, l'effectif de population calculé par Henripin et Péron est certainement plus près de la réalité que celui avancé par le recensement lui-même. En se servant des anciennes estimations de population basées sur les données des recensements, Henripin avait calculé, pour la décennie 1761-1770, un taux brut de natalité de $65,2 \%{ }^{31}$, taux phénoménal qui impliquait une augmentation de la fécondité légitime au début du régime anglais. Avec les nouvelles estimations de population, Henripin et Péron ont calculé un taux brut de natalité de

30 Langlois, op. cit., 159-160.

31 Jacques Henripin, Tendances et facteurs de la fécondité au Canada (Ottawa, Bureau fédéral de la statistique, 1968), 5. 
$56,8 \% c^{32}$, ce qui est plus vraisemblable. Par ailleurs, un témoignage contemporain confirme ce nouveau chiffre: en effet une note ajoutée à la fin du cahier conservé aux Archives Nationales et signée par «J. Goldfrap, D[eputy] Sec[retar]y [of this Province]», évalue à 80.000 habitants la population canadienne en 1765 et spécifie qu'à l'intérieur même des paroisses recensées des habitants ont échappé au dénombrement:

....at the time the foregoing Ressencements were taken, there were many persons absent as well as others, who from Infirmities, or other reasons did not attend to take the Oaths. - It is computed by many that there are Eighty Thousand Canadian Subjects within this Province.

Dans l'état actuel de la recherche, l'évaluation de 80.300 habitants en 1765 est sans aucun doute la plus réaliste. D'autre part, quelle était l'estimation exacte de la population catholique d'après le recensement de 1765 ? En additionnant aux effectifs de la population rurale les chiffres des populations urbaine (Québec et Montréal) et indigène ("who are all Roman Catholics») avancés par le gouverneur Murray, et en y soustrayant le nombre de cinq cents protestants généralement reconnu pour ces années, nous obtenons les résultats suivants :

\section{TABLEAU 4}

Population catholique de la province de Québec d'après le recensement de 1765

\begin{tabular}{lcccccc}
\multicolumn{1}{c}{ Source } & $\begin{array}{c}\text { Population } \\
\text { rurale }\end{array}$ & $\begin{array}{c}\text { Population } \\
\text { urbaine }\end{array}$ & $\begin{array}{c}\text { Population } \\
\text { indigène }\end{array}$ & $\begin{array}{c}\text { Protes- } \\
\text { tants }\end{array}$ & Total \\
Lettre de Murray & $54.575+14.700+7.400$ & - & 500 & $=76.175$ \\
Compilation des ANQ & $55.110+14.700+7.400$ & + & 500 & $=$ & 76.710
\end{tabular}

Source: APC, MG 21, série B, vol. 8: 1; ANQ, QBC-8-1.

Ces résultats assument que tous les indigènes «living within the Limits of the Province» (Murray) étaient parfaitement encadrés par le clergé canadien et que, par conséquent, tous leurs événements démographiques étaient inscrits dans les registres paroissiaux (qui ont servi à déterminer la population catholique totale en 1765). Or, il est loin d'être assuré que c'était le cas. Le feu a détruit une

32 Henripin et Péron, loc. cit., 30. 
bonne partie des registres des missions de Caughnawaga, St-Régis et St-François-du-Lac ${ }^{33}$ et nous savons qu'une certaine fraction des indigènes n'était pas sédentaire et échappait aux contrôles de l'Eglise canadienne. La prudence nous commande donc de ne considérer, dans un but de comparaison avec la nouvelle estimation de la population catholique totale, qu'une portion, disons la moitié, de ces 7.400 indigènes. La population catholique recensée en 1765 est alors réduite aux environs de 72.700 habitants. Comme on a évalué à 80.300 habitants la population catholique totale de la province de Québec en 1765, le recensement a donc sous-dénombré cette population de 7.600 habitants, soit de $10 \%$.

Ce sous-dénombrement a-t-il été engendré dans les paroisses rurales ou dans les villes? Sans discuter immédiatement de la vraisemblance de l'estimation de 14.700 habitants pour Québec et Montréal, il est certain que les paroisses rurales y sont pour quelque chose: nous avons déjà constaté qu'une dizaine parmi celles-ci, dont les registres étaient ouverts en 1765 , ne figuraient pas dans la liste des paroisses recensées. Des recherches ultérieures pourront mieux localiser ce sous-dénombrement en rapportant les naissances de 1765, que Tanguay a compilées par comté, à la population des paroisses regroupées selon les mêmes comtés ${ }^{34}$.

\section{Les versions imprimées du recensement}

Les documents manuscrits que nous venons de présenter et de critiquer n'ont pas été souvent consultés et utilisés par les chercheurs. La plupart de ceux qui ont observé l'évolution démographique de la province de Québec à cette époque se sont plutôt servis de l'une ou l'autre des deux versions publiées. Aussi croyonsnous nécessaire de présenter brièvement ces publications et de souligner les erreurs qui s'ajoutent à celles du document original.

\section{1 - Le RÉsumé PubliÉ PAR LE BUREAU de la STATISTIQUe du CANADA}

Dans le volume IV du Recensement du Canada - 1870-71, consacré à une revue rétrospective de la population canadienne par

${ }^{33}$ Raymond, loc. cit., 144; J.-A. Maurault, Histoire des Abénakis depuis 1605 jusqu'à nos jours (s.1., 1866), 492.

${ }_{34}$ Des travaux préliminaires effectués par Hubert Charbonneau concluent au sous-dénombrement évident (révélé par les taux de natalité exagérés) de certains comtés: Laprairie (oubli de Laprairie, Caughnawaga, St-Constant et St-Philippe), Maskinongé (oubli de Louiseville), Hochelaga, Chambly, St-Hyacinthe, Berthier (oubli de St-Cuthbert), L'Assomption (oubli d'Oka), etc. 
les recensements, on publia les résultats récapitulatifs du recensement de 1765 pour les cent dix paroisses qui avaient été dénombrées. Un premier tableau ${ }^{35}$ rassemble les données démographiques et les classe selon les catégories suivantes: Ménages, Sexes (M., F.), Mariés et Veuvage (M., F., Total), Enfants et non-mariés (M., F., Total). Tout en étant plus claire que celle du manuscrit, cette catégorisation a cependant l'inconvénient d'éliminer les renseignements sur l'âge, la domesticité et la présence d'étrangers. Dans un second tableau ${ }^{36}$, les statistiques agricoles sont reproduites selon les mêmes catégories que le manuscrit des Archives Nationales du Québec.

La colonne (4) de notre tableau 2 fournit les totaux de la population de chaque paroisse tels que publiés. La comparaison de ces chiffres avec ceux des colonnes (1) et (2) est très révélatrice. Elle met d'abord en évidence la divergence des effectifs avancés par chaque source. Cette divergence est plus prononcée avec la version corrigée du cahier des Archives Nationales qu'avec sa version brute. En effet, dans le premier cas, les résultats ne coïncident que pour six paroisses sur trente-six et l'écart global est de 3,5\%, tandis que dans le deuxième cas les résultats coïncident pour trente-huit paroisses sur cent dix et l'écart global n'est que de 1,5\% $\%^{37}$. Ces comparaisons établissent que le Bureau de la statistique du Canada a fondé l'effectif total de la population de plus du tiers des paroisses de la province de Québec sur les résultats bruts, non préalablement épurés de leurs erreurs d'addition, contenus dans le cahier manuscrit du recensement de 1765 .

Pour les deux autres tiers des paroisses, l'écart des résultats publiés avec ceux de la version brute est souvent minime. Mais dans le cas de sept paroisses, la différence est notable: la population de la paroisse Château-Richer a subi une hausse de $62,8 \%$, celle de Sorel de 49\%, celle de Baie des Chaleurs - Bonaventure de 25,9\%, celle de St-Augustin de 23,8\%, celle de St-Charles de 11,8\%, celle de St-Henri de 10,1\% et celle de la Rivière St-Charles (dépendant de Charlesbourg) a subi une baisse de $12,1 \%$. Où les statiticiens fédéraux ont-ils puisé ces nouveaux résultats? Sûrement pas dans les feuillets (rôles) du recensement, si l'on se fie aux données du tableau

\footnotetext{
35 Canada, Recensement du Canada - 1870-71, volume IV: Recensements du Canada. 1665 à 1871 (Ottawa, 1876): 64-65.

36 Ibid. : 66-67.

37 La comparaison avec les chiffres du tableau 3 pour les sept paroisses du gouvernement de Trois-Rivières va aussi dans le même sens.
} 
3. Peut-être s'agit-il de corrections qu'ils ont eux-mêmes apportées aux résultats bruts? C'est possible dans les cas des paroisses dont les effectifs ont été les plus transformés. Par exemple, dans cette version publiée, la paroisse de Sorel a été dédoublée: "partie sur le fleuve» et partie "dans l'intérieur». Cette dernière partie reproduit fidèlement les résultats consignés dans le cahier des Archives Nationales du Québec, tandis que les chiffres de la "partie sur le fleuve» n'apparaissent pas sur le manuscrit. Peut-être les statisticiens ont-ils voulu corriger un mécompte ou un oubli du recenseur, mais en se basant sur quoi? Nous l'ignorons.

Outre ces «corrections», inexpliquées et inexplicables, l'autre originalité de ce résumé est d'avoir fourni une évaluation de la population des villes de Québec et de Montréal, lesquelles, nous le savons, n'ont pas été recensées en 1765 . Les auteurs ont d'abord accepté le chiffre de 14.700 habitants, total des effectifs de ces deux villes présenté par le gouverneur Murray à Lord Shelburne ${ }^{38}$. Ils ont ensuite effectué le partage entre les deux villes en s'appuyant «sur les proportions des recensements antérieurs " ${ }^{39}$. Ils ont ainsi attribué une population de 8.967 habitants $(61 \%)$ à Québec et de 5.733 habitants (39\%) à Montréal.

L'évaluation de 14.700 habitants pour l'ensemble des deux villes est-elle réaliste? En rapportant le nombre annuel moyen de naissances catholiques survenues à Québec et à Montréal pendant les années 1764,1765 et 1766 (677) à cette évaluation de 14.700 habitants ${ }^{40}$, nous calculons un taux brut de natalité de $46,1 \%$. Ce taux est certes inférieur à celui de $56,8 \%$, affecté par Henripin et Péron à l'ensemble de la population catholique de la province de Québec pour la décennie 1761-1770, mais cet écart peut être aisément attribuable à une proportion plus grande de célibataires, liés au commerce et au gouvernement, qui habitaient les villes. L'évaluation de 14.700 habitants pour l'ensemble de la population urbaine est donc vraisemblable.

Qu'en est-il maintenant du partage de cet effectif entre les deux villes? En calculant les statistiques moyennes du mouvement naturel

38 Les statisticiens ne donnent pas la lettre de Murray comme référence, mais plutôt «un mémoire trouvé dans les archives de la fabrique du Cap-Santé» (ibid. : xxxviii), lequel, sans doute, s'appuie sur la lettre du gouverneur.

39 Ibid.: 68

40) Le nombre de naissances protestantes étant probablement très faible, notre taux s'en trouve très peu modifié. 
des populations de Québec et de Montréal pour les années 1764, 1765 et 1766 , nous parvenons aux résultats suivants:

\section{TABLEAU 5}

Répartition des événements démographiques entre les villes de Québec et Montréal, 1764-1765-1766

\begin{tabular}{|c|c|c|c|c|}
\hline Villes & Naissances & Mariages & Décès & $\begin{array}{l}\text { Total des } \\
\text { événements }\end{array}$ \\
\hline Québec & $47,3 \%(320)$ & $44,4 \% \quad(48)$ & $44,7 \%$ (245) & $46 \% \quad(613)$ \\
\hline Montréal & $52,7 \%(357)$ & $55,6 \% \quad(60)$ & $55,3 \%(303)$ & $54 \% \quad(720)$ \\
\hline Total & $100 \%(677)$ & $100 \%$ & $100 \%$ & $100 \%$ \\
\hline
\end{tabular}

Source: Canada, Recensement du Canada - 1870-71, volume V: Recensements $d u$ Canada. 1608 à 1876 (Ottawa, 1878): 207-208.

Si nous partons de l'hypothèse que l'intensité de la natalité, de la nuptialité et de la mortalité était la même pour Québec et Montréal et que l'enregistrement des événements démographiques était de même qualité dans les deux villes, la population urbaine de la province de Québec serait répartie dans une proportion de 54\% à Montréal et de $46 \%$ à Québec, et non, comme l'ont évaluée les statisticiens fédéraux, de $39 \%$ à Montréal et $61 \%$ à Québec. L'effectif de la population de Montréal aurait donc été de 7.938 habitants (et non de 5.733) et celui de Québec de 6.762 habitants (et non de 8.967) ${ }^{41}$.

Comme ces nouveaux chiffres reposent sur plusieurs hypothèses discutables et dérivent du seul critère de la répartition des événements démographiques, ils ne sont pas irréfragables, d'autant plus qu'ils contredisent non seulement plusieurs recensements, mais aussi certains témoignages contemporains ${ }^{42}$. Ils jettent toutefois un éclairage nouveau sur le problème de l'équilibre des forces démographiques

41 Une étude récente de Louise Dechêne a souligné l'importance de la banlieue rurale à l'intérieur des limites de la paroisse Notre-Dame-de-Montréal. Grâce à quatre dénombrements de domiciles dressés en $1697,1731,1741$ et 1781 , elle a pu évaluer cette population agricole assez stable à un peu moins de mille habitants. La population urbaine proprement dite de l'île de Montréal ne devait donc pas dépasser en 1765, selon nos propres chiffres, sept mille habitants. Louise Dechêne, "La croissance de Montréal au XVIIIe siècle", Revue d'histoire de l'Amérique française, 27, 2 (septembre 1973): 163-165.

42 Ibid.: 164, et E.R. Adair, "The Evolution of Montreal under the French Regime», The Canadian Historical Association, Report 1942: 34. 
entre les villes de Québec et de Montréal au XVIII ${ }^{\mathrm{e}}$ siècle et ils ne sont pas sans évoquer l'épineuse question de l'émigration des Canadiens au moment de la conquête. Mais seuls les progrès de la recherche nous permettront éventuellement de déterminer dans quelle mesure l'estimation traditionnelle des populations urbaines a pu être faussée par ce phénomène d'émigration.

\section{2 - LA TRANSCRIPTION DE L'ARCHIVISTE P.-G. ROY}

Le département du Secrétaire de la province de Québec publia en 1937 la transcription du cahier manuscrit du recensement de 1765 que l'archiviste Pierre-Georges Roy avait découvert par «un heureux hasard [...] dans les Archives de la province de Québec ${ }^{43}$ ».

Il s'agit d'une copie très fidèle du document que nous avons précédemment décrit et critiqué. Précisons toutefois que nous n'avons vérifié l'exactitude de cette transcription que pour chaque total des colonnes relatives aux données démographiques, pour chaque paroisse. Nous ne pouvons donc pas attester l'exactitude de la reproduction des informations relatives à chaque ménage en particulier, ni de celle des données agricoles.

Des lacunes que nous avons pu relever dans cette transcription, mentionnons d'abord que Roy n'a reproduit les sous-totaux cumulatifs que pour quelques paroisses (e.g. Lachine, St-Laurent, Pointe-Claire, Batiscan...); cette omission, voulue peut-être parce que les erreurs d'addition commises par le compilateur seraient devenues trop manifestes, oblige donc l'observateur soucieux d'expliquer toutes ces erreurs à recourir au manuscrit. Puis, à de très rares endroits, à la vue de ce qui semblait des erreurs grossières d'addition, Roy a substitué certains résultats. Par exemple, dans la paroisse de St-Mathias, à la colonne des domestiques mâles audessus de 15 ans, le cahier manuscrit avait additionné $4+0=10$; au lieu de 10, Roy a transcrit $4^{44}$. Autre exemple, dans la paroisse de Varennes, à la même colonne, le compilateur du manuscrit avait totalisé $26+6=31$; Roy a substitué $32{ }^{45}$. Bien que ces corrections étaient justifiables, nous croyons que l'archiviste doit reproduire fidèlement un document, et non le corriger sans même en avertir le lecteur. Faute plus grave, en une occasion, Roy a voulu rectifier

43 Rapport de l'Archiviste de la province de Québec pour 1936-1937, Département du Secrétaire de la province (Québec, 1937): 1.

44 Ibid.: 77.

45 Ibid.: 113. 
une somme qui était fausse; ce faisant, il a inscrit un nouveau chiffre qui est beaucoup plus éloigné du résultat juste que celui fourni par le compilateur du manuscrit ${ }^{46}$.

Bref, pour qui veut travailler avec les données du recensement de 1765 , il n'y a aucun avantage à recourir à ces deux publications qui n'ont pas été faites avec méthode et dont les lacunes s'ajoutent à celles du document original.

L'examen critique des résultats du recensement de 1765 , conjugué à l'utilisation des statistiques du mouvement naturel de la population, nous ont permis de conclure qu'environ $10 \%$ de la population catholique de la province de Québec, répartis principalement à l'intérieur d'une dizaine de paroisses rurales, n'avaient pas été recensés en 1765 .

Ces résultats n'ont pas la prétention d'être définitifs. Ils reposent sur un certain nombre d'hypothèses qui demeurent contestables. Néanmoins nous croyons avoir réussi à prouver que le recensement de 1765 était loin d'être parfait et qu'un usage inconsidéré de ses résultats pouvait mener à la négation d'importantes réalités démographiques.

Trop souvent, dans le passé, les historiens ont utilisé les documents quantitatifs sans s'interroger sur leur exactitude. C'est ainsi que Fernand Ouellet a utilisé le recensement de 1765 pour étayer ses affirmations concernant la «ruralisation» de la population après la conquête et certains développements de la production agricole ${ }^{47}$. Quiconque a pris conscience de la multitude d'erreurs que renferment nos recensements anciens, dont celui de 1765, conviendra de la fragilité de telles conclusions.

Aussi est-il nécessaire de rappeler que la critique des sources, tant négligée dans le passé, est incontestablement l'un des prérequis essentiels à la connaissance de la réalité historique.

46 Paroisse de Varennes (ibid.: 113), colonne des enfants femelles: le compilateur avait additionné 325 ; Roy a écrit 322 ; la réponse juste est 323.

47 Fernand Ouellet, Histoire économique et sociale du Québec, 1760-1850 (Montréal, Fides, 1966), 81. 